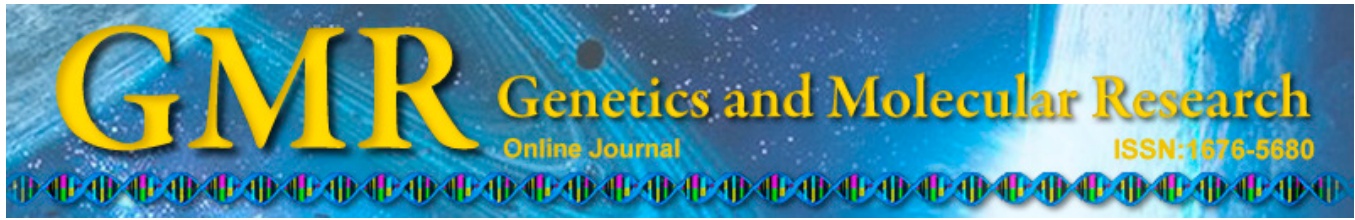

\title{
Ectopic expression of the BoTFL1-like gene of Bambusa oldhamii delays blossoming in Arabidopsis thaliana and rescues the $t f 1$ mutant phenotype
}

\author{
H.Y. Zeng ${ }^{1,2}$, Y.T. Lu' ${ }^{1}$ X.M. Yang ${ }^{1}$, Y.H. Xu ${ }^{3}$ and X.C. Lin ${ }^{1}$ \\ ${ }^{1}$ The Nurturing Station for the State Key Laboratory of Subtropical Silviculture, \\ Zhejiang Agriculture and Forestry University, Lin'an, Hangzhou, \\ Zhejiang, China \\ ${ }^{2}$ Lishui City Liandu District Forestry Bureau, Lishui, Zhejiang, China \\ ${ }^{3}$ School of Agriculture and Food Science, \\ Zhejiang Agriculture and Forestry University, Lin'an, Hangzhou, Zhejiang, China \\ Corresponding author: X.C. Lin \\ E-mail: 1xc@zafu.edu.cn
}

Genet. Mol. Res. 14 (3): 9306-9317 (2015)

Received January 21, 2015

Accepted April 8, 2015

Published August 10, 2015

DOI http://dx.doi.org/10.4238/2015.August.10.11

\begin{abstract}
TERMINAL FLOWER1 (TFL1) homologous genes play major roles in maintaining vegetative growth and inflorescence meristem characteristics in various plant species; however, to date, the function of the bamboo TFL1 homologous gene has not been described. In this study, a TFL1 homologous gene was isolated from Bambusa oldhamii and designated as BoTFL1-like. Phylogenetic analysis of TFL1 homologous genes revealed that BoTFL1-like shared more than $90 \%$ identity with the TFL1 genes of other Gramineae. RT-PCR analysis showed that the expression level of BoTFL1-like in floral buds was almost 3.5 times higher than in vegetative buds. In $35 S: \because$ BoTFL1like transgenic Arabidopsis thaliana plants, the time of flowering was significantly delayed by 5 to 9 days, and development of floral buds
\end{abstract}


and sepals was severely affected compared to wild type Arabidopsis plants. This suggests that the BoTFL1-like gene may play roles in flowering time and flower morphological structure in B. oldhamii. The BoTFL1-like gene driven by the $35 \mathrm{~S}$ promoter almost fully rescued the phenotype of the tfll mutant apart from the number of rosette inflorescences, indicating that the function of BoTFL1-like was similar to TFL1 in Arabidopsis. We conclude the TFL1 gene function has been conserved between $B$. oldhamii and A. thaliana.

Key words: Bambusa oldhamii; Gene cloning; Sequence analysis; TERMINAL FLOWER1; Transgenic Arabidopsis thaliana

\section{INTRODUCTION}

The transition from vegetative to reproductive growth is a crucial developmental switch in flowering plants. Many genetic and molecular components underlying floral transition have been characterized in Arabidopsis thaliana (Hayama and Coupland, 2004). The TFL1 (TERMINAL FLOWER1) gene, which has an important role in flowering transition, controls inflorescence meristem identity through inhibition of the expression of flower meristem identity genes (Ohshima et al., 1997; Abdullah et al., 2009; Xi et al., 2010; Tahery et al., 2011; Wang et al., 2011).

TFL1 is a member of the FT/TFL1 gene family in Arabidopsis that includes FLOWERING LOCUST $(F T)$ and four related genes, namely, TWIN SISTER OF FT, BROTHER OF FT AND TFL1, ARABIDOPSIS THALIANA CENTRORADIALIS HOMOLOGUE, and MOTHER OF FT AND TFL1 (Kobayashi et al., 1999). The FT/TFL1 gene family encodes proteins with similarity to phosphatidyl ethanolamine binding proteins (PEBPs); however, the genes vary in their roles in the regulation of flowering transition and act as either promoters or repressors depending on the key nucleotide of the protein function domain (Banfield et al., 1998; Hengst et al., 2001; Kikuchi et al., 2009).

TFL1 has been proposed to be antagonistic with $L F Y$ (LEAFY), AP1 (APETALA1), and other floral meristem genes, and this antagonism is manifested in their complementary expression patterns and phenotypic effects (Bowman et al., 1993; Parcy et al., 2002). The phenotypes produced by a reduction in TFL1 activity are similar to the effects of the constitutive expression of $L F Y$ and $A P 1$ (Ratcliffe et al., 1999). The expression of the floral meristem identity genes can be inhibited by the shoot meristem identity gene TFL1, which encodes a protein that is likely involved in signal transduction, thereby preventing flowering (Shannon and Meeks-Wagner, 1991; Schultz and Haughn, 1993). By contrast, the activity of TFL1 can be suppressed by $L F Y$, and AP1. TFL1 expression is not detected in transgenic Arabidopsis carrying either a $35 S:: L F Y$ construct or a $35 S: \because A P 1$ construct (Liljegren et al., 1999; Ratcliffe et al., 1999).

Bambusa oldhamii belongs to the bamboo family, the largest member of the Poaceae. It is well-known for its edible shoots and green timber, and is one of the most economically important forest resources in tropical and subtropical countries (Hsu et al., 2000; Lin et al., 2009). The majority of bamboos do not flower until they are approximately 30 - to 60 -yearsold. Bamboo flowering is unpredictable, gregarious, and the plants usually die after flowering. This not only reduces local income, but also causes ecological and environmental problems (Daniel and Janzen, 1976; Lin et al., 2009). Therefore, there are compelling reasons to study 
the genetics of the flowering mechanism in bamboos. A number of factors may influence flowering in bamboo such as environment, nutrition, climate, and physiological status, and several hypotheses have been proposed to explain the pattern of flowering (Daniel and Janzen, 1976; Gielis et al., 1999; Franklin, 2004). In recent years, there has been increasing focus on identifying and characterizing the genes that regulate flowering in bamboo species. Thus, DIMADS1-18 full-length cDNA sequences were isolated from the panicles of Dendrocalamus latiflorus and it was found that overexpression of DIMADS8 or DIMADS18 in Arabidopsis causes leaf curl and an early flowering phenotype (Tian et al., 2005, 2006). Two AP1/SQUA-like MADS-box genes, PpMADS1 and PpMADS2 were isolated from Phyllostachys praecox during floral transition; in Arabidopsis, overexpression of these genes significantly promotes early flowering through upregulation of $A P 1$ (Lin et al., 2009). Two cDNA libraries have been established from flower buds and vegetative shoots of $B$. oldhamii. From these libraries, 4470 and 3878 ESTs have been annotated, respectively, and many flowering-related genes have been identified (Lin et al., 2010). Recently, a draft sequence of the genome of moso bamboo (Phyllostachys heterocycla) was produced and a large number of candidate flowering genes were identified and shown to be transcription factor genes, heat shock protein genes or other stress-responsive genes (Peng et al., 2013). Although substantial advances have been made, a complete description of the genetic regulation of flowering in bamboo species is still not available.

In this study, we isolated a full-length cDNA of a TFL1-like gene from B. oldhamii and investigated its expression pattern. We generated CaMV 35S::BoTFL1-like transgenic Arabidopsis plants to assay the putative characteristics and function of this gene. Our results provide important insights into the mechanism of flowering in B. oldhamii.

\section{MATERIAL AND METHODS}

\section{Plant materials and growth conditions}

Bamboo plants used for gene cloning and expression analysis were obtained from the bamboo tissue culture room of Zhejiang Forestry Academy, the Nurturing Station for the State Key Laboratory of Subtropical Silviculture, Zhejiang Agriculture and Forestry University; this is the same source as used in a previous study (Lin et al., 2010). Vegetative shoots and floral buds were collected from plants subcultured for two weeks, and used to obtain RNA for the real-time quantitative reverse transcription polymerase chain reaction (RT-PCR) analysis. A. thaliana wild type (ecotype Columbia; seeds from ABRC, Ohio State University, Columbus, OH, USA) and transgenic plants were grown on Murashige and Skoog (MS) 0.5X agar medium. Hygromycin B (50 mg/L) was used to select transgenic Arabidopsis plants on MS $0.5 \mathrm{X}$ agar medium. Wild type and transgenic Arabidopsis plants were grown in a temperaturecontrolled room at $22^{\circ} \mathrm{C}$ under long day ( $16 \mathrm{~h}$ light) conditions.

Homozygous tfll-13 mutant plants (induced by EMS mutagenesis) were purchased from TAIR (serial number CS6237); this mutant carries Lys instead of Glu at position 87.

\section{RNA isolation}

The tissues described above were placed in liquid nitrogen, ground into powder, and total RNA was extracted using an RNAiso plus kit (Takara, Japan) according to manufacturer instructions. 


\section{Cloning of BoTFL1-like from B. oldhamii}

To amplify TFL1-like sequences, a pair of primers (F1 and R1) was designed based on the conserved DNA sequence of TFL1 homologous genes of graminaceous plants. We performed 3'-rapid amplification of cDNA ends (3'-RACE) and 5'-RACE by using a BD SMART $^{\text {TM }}$ RACE cDNA Amplification Kit (Clontech, Mountain View, CA, USA) according to the manufacturer instructions. For the 3'-RACE, amplification was performed with the $3^{\prime}$ abridged universal amplification primer (3'-AUAP) and two gene-specific primers (F1 and F2). For the 5'-RACE, 5 ' specific primers (R1 and R2), the abridged anchor primer, and the 5'AUAP were used. The full-length cDNA of BoTFL1-like was obtained by PCR amplification using the forward primer ORFF and reverse primer ORFR. The primers used in this study are listed in Table 1.

Table 1. Sequences of the primers used in this study.
\begin{tabular}{lll}
\hline Primer & Sequences $\left(5^{\prime} \rightarrow 3^{\prime}\right)$ & Description \\
\hline F1 & ATGACAGACCCTGATGTGCCA & Forward primer for conserved sequence and 3'-RACE \\
R1 & CCTTCGCTTCTGCTTGAAGAG & Reverse primer for conserved sequence and 5'-RACE \\
F2 & AAAGCCCAACATCGGTATCCAC & Nested gene-specific primer for 3'-RACE \\
R2 & GACAATCCAGTGAAGGTGCTCC & Nested gene-specific primer for 5'-RACE \\
ORFF & ATGTCTAGGTCTGTGGAGCCTC & Forward primer for ORF \\
ORFR & TCAGCGTCTCCTGGCAGCAGTC & Reverse primer for ORF \\
RT-F & CCTTCACATTGGTTATGACAGA & Forward primer for real-time quantitative RT-PCR \\
RT-R & CAGCAGTCTCTCTCTGAGCATT & Reverse primer for real-time quantitative RT-PCR \\
actinF & GAGCGAGAAATTGTCAGGGA & Actin primer as internal control, forward \\
actinR & GATGGCTGGAAGAGGACCT & Actin primer as internal control, reverse \\
\hline
\end{tabular}

\section{Phylogenetic analysis}

The amino acid sequences of TFL1 homologues from various plant species were obtained from GenBank (http://www.ncbi.nlm.nih.gov/) and aligned with DNAMAN 6.0. A phylogenetic tree based on the complete sequences was generated using PhymL (http://www.atgc-montpellier. fr/phyml/) and constructed using the neighbor-joining method. Bootstrap values were set to 1000 .

\section{Real-time quantitative RT-PCR}

Real-time quantitative PCR was performed using the SYBR Premix Ex Taq kit (Takara) based on manufacturer instructions. The specific primers were RTF and RTR (Table 1), and the actin primers were actinF and actinR (Table 1). Each cDNA template was used in three replicates. All reactions were performed with the CFX96 Real-Time PCR Detection System (Bio-Rad), and data were analyzed with the CFX96 system software.

\section{Construction of expression vector and Arabidopsis transformation}

The open reading frame (ORF) of the BoTFL1-like cDNA was amplified using Pfu DNA Polymerase (BoCai Biotechnology, Shanghai, China) with forward primer ORFF and reverse primer ORFR, and inserted into a pMD20-T vector (Takara). Both pMD20-T vector and pCAMBIA1301 vector were digested with $K p n I$ and $X b a \mathrm{I}$ (Takara) and then the BoTFL1- 
like ORF was cloned into the pCAMBIA1301 vector that contains the cauliflower mosaic virus (CaMV) $35 \mathrm{~S}$ promoter. The resulting recombinant plasmid was then transformed into Agrobacterium tumefaciens strain GV3101 by electroporation using the Gene Pulser MXcell electroporation system (Bio-Rad, USA).

Arabidopsis plant transformation was carried out using the floral dipping method (Clough and Bent, 1998). Transgenic plants were screened on 1/2 MS agar medium supplemented with hygromycin B $(50 \mathrm{mg} / \mathrm{L})$ before they were transplanted into soil. The flowering time and rosette leaf numbers of three T3 transgenic lines were counted when the first flower started to open on the main inflorescence, and floral structure was also screened. Each line had 30 repetitions. Insertion of the BoTFL1-like gene into the Arabidopsis genome was confirmed by PCR analysis using the primers ORFF and ORFR (Table 1).

\section{RESULTS}

\section{BoTFL1-like is a homolog of TFL1}

A 186-bp fragment of the TFL1 gene was amplified from B. oldhamii based on the conserved TFL1 sequence of graminaceous plants. Using gene-specific primers, a homologue of TFL1 was isolated from B. oldhamii using 3' and 5'-RACE and was designated as BoTFL1like. DNA sequence analysis showed that the entire cDNA length was $833 \mathrm{bp}$ and the genome DNA sequence was 1225 bp containing a complete ORF that encoded a polypeptide of 173 amino acids, and had an 85-bp untranslated 5' region and a 226-bp 3' untranslated region (Figure 1). Comparison of the BoTFL1-like gene and cDNA sequences showed that the BoTFL1like gene had 4 exons and 3 introns (Figure 2A). Amino acid sequence alignment revealed that the BoTFL1-like protein had similar conserved regions as TFL1 proteins of other species (Figure 2B). Secondary structure analysis using SOPMA indicated that the putative BoTFL1-like protein contained an alpha helix $(16.18 \%)$, a rich random coil $(53.73 \%)$, a beta turn structure $(5.78 \%)$, and an extended strand (24.28\%; Figure 3A). Amino acid sequence alignment with PEBP family members showed that the BoTFL1-like protein contained the key functional site and ligand binding site, and that amino acid residues $71 \mathrm{D}, 85 \mathrm{H}, 87 \mathrm{H}, 110 \mathrm{~S}, 111 \mathrm{P}, 112 \mathrm{~K}, 118 \mathrm{H}$, and $120 \mathrm{~F}$ could be involved in the formation of the ligand binding site (Figure 3B).

The sequences of TFL1 homologs from over 20 plant species were downloaded from NCBI to investigate the phylogenetic relationship of the BoTFL1-like protein with TFL1 proteins of other species; a phylogenetic tree was constructed using PhymL. This tree showed that the BoTFL1-like protein clustered with grass species, and had a 91\% identity with barley and ryegrass, and $90 \%$ with corn and rice (Figure 4 ). The data supported the interpretation that the BoTFL1-like gene is a TFL1 homolog.

\section{Expression level of BoTFL1-like in floral and vegetative buds}

To determine if BoTFL1-like transcripts accumulated in vegetative and floral tissues, BoTFL1-like RNAs were quantified using RT-PCR. The level of BoTFL1-like transcripts in floral buds was almost 3.5-fold higher than in vegetative buds (Figure 5), indicating that BoTFL1-like gene expression was lower in vegetative stage tissues and significantly increased in reproductive stage tissues. Thus, the BoTFL1-like gene may be involved in maintaining reproductive growth. 


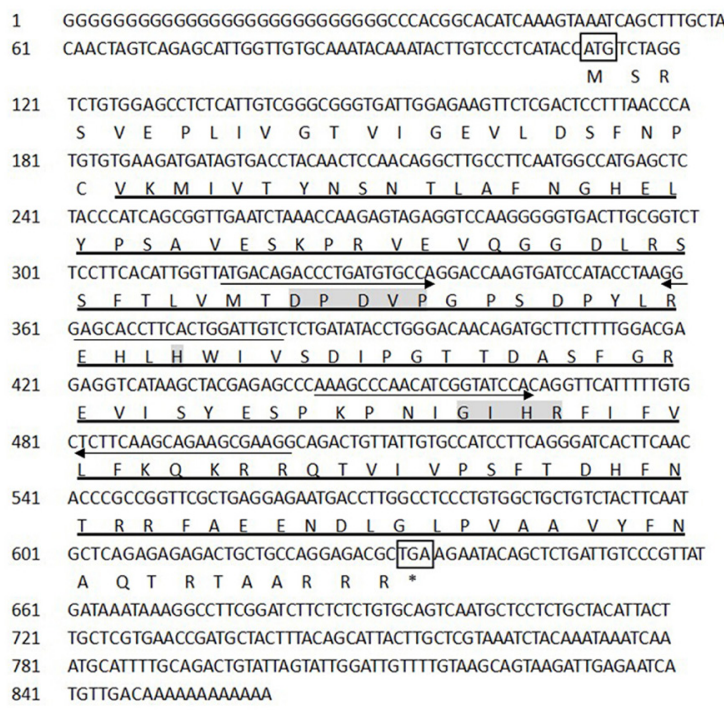

Figure 1. Nucleotide sequence of the BoTFL1-like gene and its predicted amino acid sequence. Initiation and stop codons are marked by black boxes, and PEBP regions are underlined. Important protein functional modules in TFL1 are indicated by gray shading.

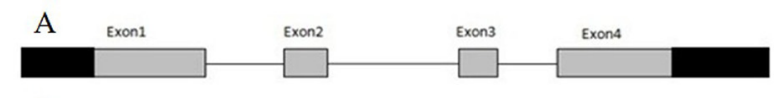

$\mathrm{B}$

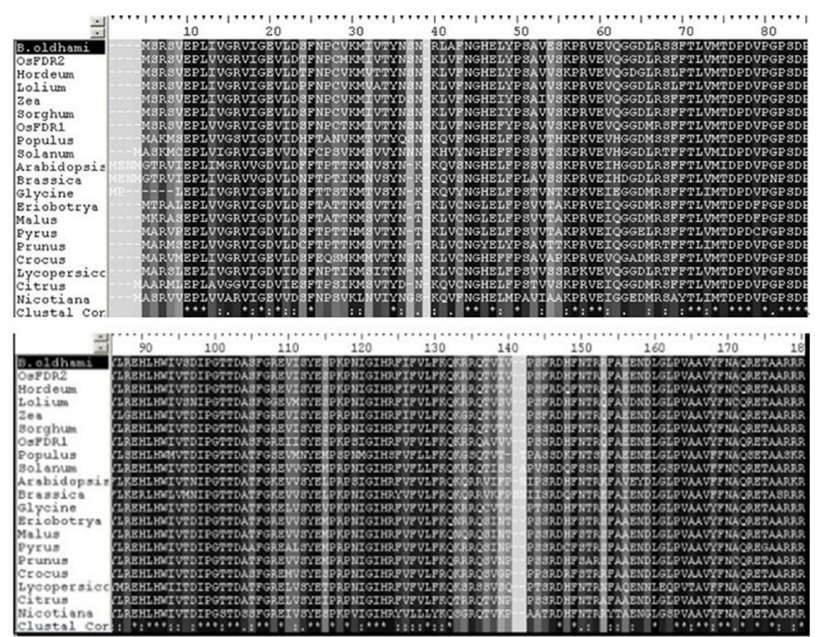

Figure 2. Genomic organization of the BoTFL1-like gene and protein sequence similarities. (A) Genomic organization of BoTFL1-like showing untranslated (black boxes) and translated (gray boxes) regions. (B) Alignment of partial amino acid sequences of TFL1 homologs. GenBank accession Nos. are: Hordeum vulgare (DQ539338), Lolium perenne (AF316419), Oryza sativa (AF159882), Zea mays (NM001112489), Sorghum bicolor (XM002450238), Vitis vinifera (AF378127), Cucumis sativus (AB383155), Arabidopsis thaliana (ATU77674), Glycine max (EF532597), Picea abies (EF633467), Impatiens balsamina (AJ888756), Aquilegia formosa (DQ286962), Pisum sativum (AY343326), Populus trichocarpa (DQ310725), Vicia faba (ABP73382), Solanum tuberosum (DQ307621), Gossypium hirsutum (EU026443), Pyrus communis (AB162042), and Eriobotrya japonica (AB162051). 
A

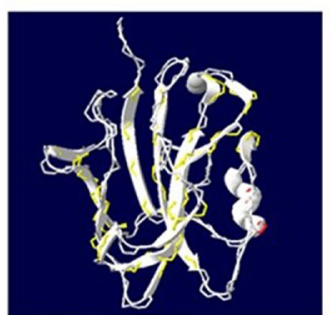

MSRSVEPLIVGRVIGEVLDSFNPCVKMIVT YNSNRLAFNGHELYPSAVESKPRVEVQGGDL RSSFTLVMTDPDVPGPSDPYLREHLHWIVSDIPGTTDASFGREVIS YESPKPNIGIHRFIFVL FKQKRRQTVIVPSFRDHFNIRRFAEENDLGLPVAAVYFNAQRETAARRR

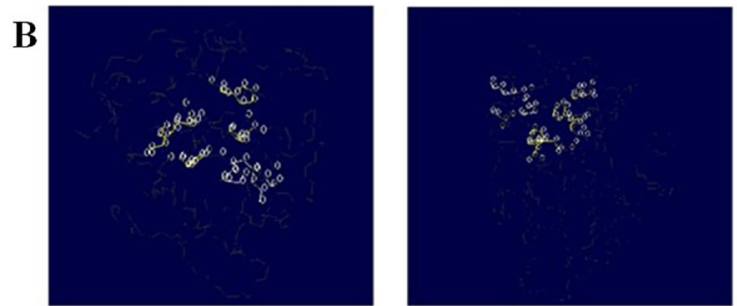

MSRSVEPLIVGRVIGEVLDSFNPCVKMIVTYNSNRLAFNGHELYPSAVESKPRVEVQGGDL

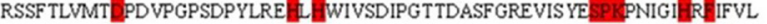
FKQKRRQTVIVPSFRDHFNTRRFAEENDLGLPVAAVYFNAQRETAARRR

Figure 3. Secondary and tertiary structure predictions for the BoTFL1-like protein. (A) Secondary structure prediction. Alpha helix is shown in red and the beta turn in yellow. (B) Functional site predictions. Amino acid side chains involved in the formation of functional sites are marked by white circles.
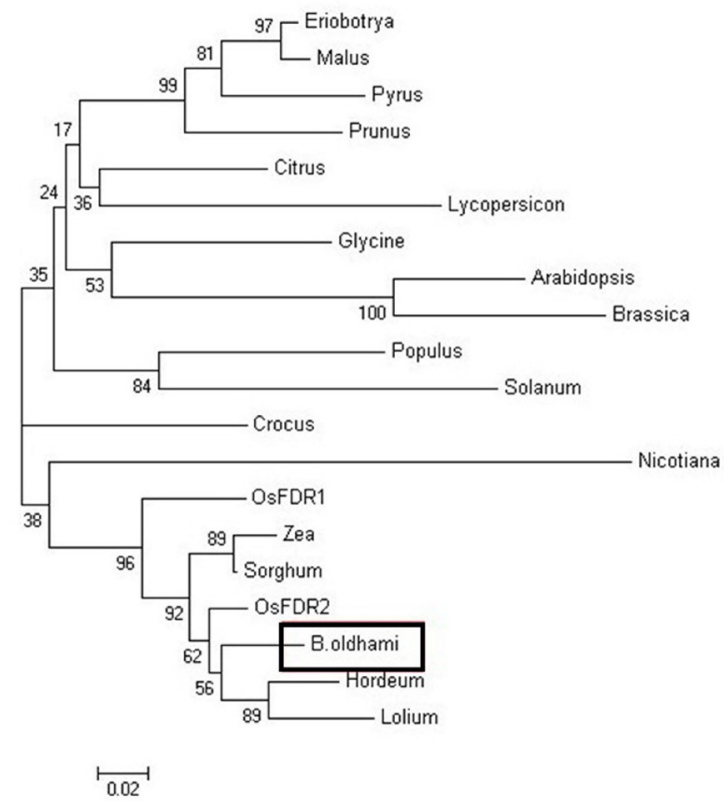

Figure 4. Phylogenetic analysis of TFL1 amino acid sequences from different plant species using PhymL. The BoTFL1-like protein is indicated by the black box. 


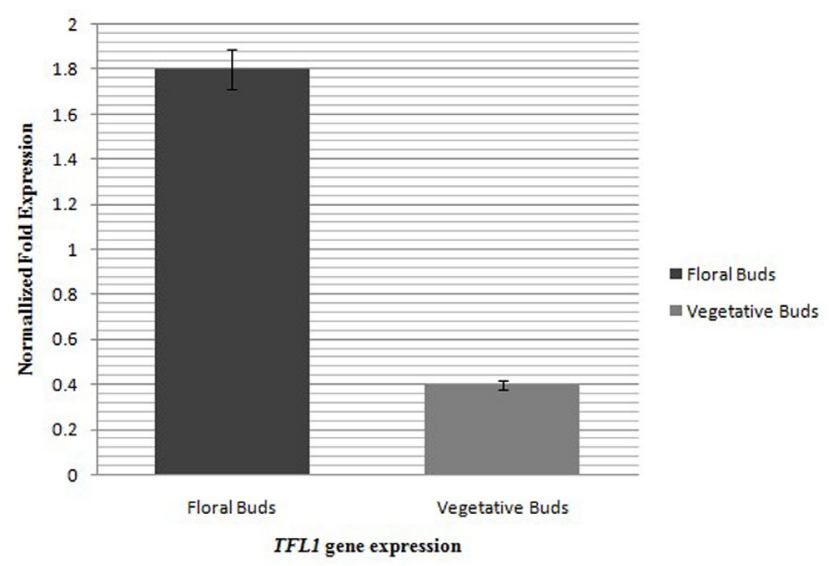

Figure 5. Real-time PCR analysis of the relative expression of BoTFL1-like gene. BoTFL1-like gene expression in floral buds is shown using black shading, while that in vegetative buds is shown using gray shading.

\section{Ectopic expression of the BoTFL1-like gene delays flowering in Arabidopsis}

To determine the potential role of BoTFL1-like gene in floral development, 35S::BoTFL1-like transgenic Arabidopsis plants were produced using Agrobacterium-mediated transformation. Twenty independent hygromycin-resistant $\mathrm{T} 1$ plants were identified and most showed a delay in flowering time compared to wild type plants. Three independent T3 homozygous transgenic lines were selected for analysis of their phenotypic variations. The flowering time of T3 transgenic plants was significantly delayed by 5 to 9 days compared to the wild type Arabidopsis (Figure 6A). In addition, the floral buds and sepals of transgenic Arabidopsis were slightly abnormal (Figure 6B, C): the transgenic sepals spread out

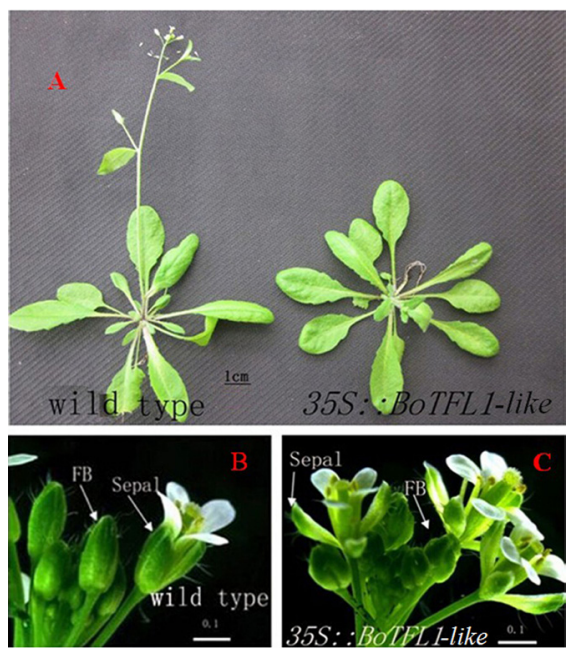

Figure 6. Comparison of transgenic and wild type Arabidopsis plants. (A) Wild type Arabidopsis (left side) blossoms at 35 days; 35S: :BoTFL1-like transgenic Arabidopsis (right side) begins to bolt after 35 days. (B) Wild type Arabidopsis flowers have sepals tightly associated with the petals, and the buds are ellipsoidal. (C) 35 S::BoTFL1-like transgenic flowers have sepals that grow out like petals, have four grooves in ellipsoid-shaped buds, and smaller buds. 
like petals, and the transgenic floral buds were small and four grooves appeared in the original ellipsoid-shaped buds. These effects suggest that the BoTFL1-like gene may be involved in the regulation of flowering time and of flower morphological structure in Arabidopsis.

\section{Ectopic expression of BoTFL1-like largely complements the tfl1 mutant}

To confirm the function of the BoTFL1-like gene, the 35S::BoTFL1-like vector was introduced into plants carrying the tfl-13 mutation to determine whether it could complement the mutant phenotype. The tfll-13 plants have an early flowering phenotype, a reduced plant height, an increased number of rosette inflorescences, determinate inflorescences, and the whole meristem is divided prior to flower organ determination (Figure 7A and B). Expression of BoTFL1-like cDNA in tfl1-13 mutants caused a prolongation of the vegetative stage to the length expected of wild type Arabidopsis; plant height was significantly increased, but the number of rosette inflorescences did not change significantly (Figure 7A). Moreover, the unusual flower structure of $t f l-13$ was restored to normal (Figure 7B and C). These results demonstrated that overexpression of the BoTFL1-like gene could largely rescue the effects of this Arabidopsis TFL1 mutation. Taken together, our data suggest that BoTFL1-like may have both similar and divergent functions to TFL1 in Arabidopsis.

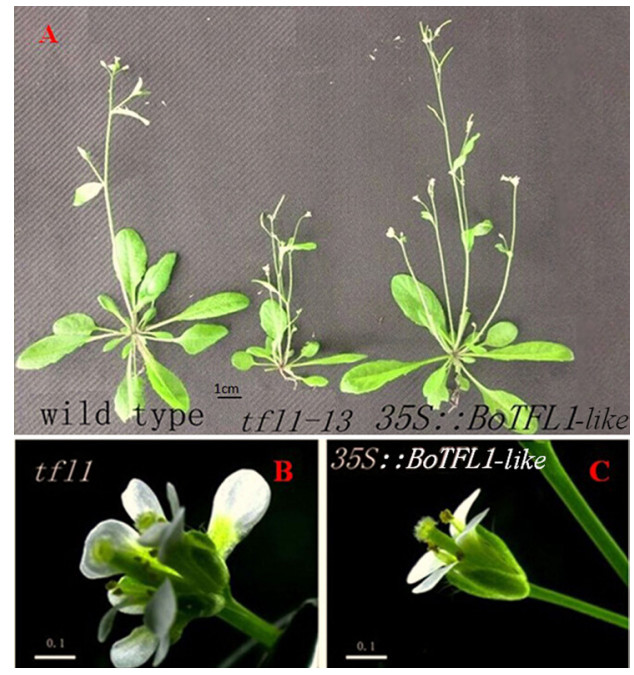

Figure 7. Comparison of floral morphologies of wild type, tfl1-13 mutant, and 35S::BoTFL1-like transgenic tfl1-13 mutant Arabidopsis plants at 35 days. (A) Wild type Arabidopsis (left side); tfl-13 mutant (middle) showing early flowering, reduced height and increased number of rosette inflorescences; 35S::BoTFL1-like transgenic mutant Arabidopsis (right side) showing phenotypic rescue for flowering time and height. (B) tfl1-13 mutant plants have an increased number of petals and abnormal flowering pattern. (C) 35S::BoTFL1-like transgenic plant shows phenotypic rescue and resembles the wild type.

\section{DISCUSSION}

In this study, the BoTFL1-like gene of the B. oldhamii was isolated and characterized. As shown in Figure 2B, it shared a high identity with TFL1 homologues of other species. 
Similarly to these TFL1 homologues, BoTFL1-like contained three introns and four exons, and had the same gene structure and intron pattern as the rice homologue OsCEN1 (Jensen et al., 2001; Lynn et al., 2004; Zhang et al., 2005; Guo et al., 2006; Kotoda et al., 2006; Argiriou et al., 2008; Sato et al., 2009; Esumi et al., 2010) (Figure 2A). BoTFL1-like encoded a polypeptide of 173 amino acids that shared high sequence identity with LpTFL1 and OsCEN1 proteins. Amino acid sequence alignment with the PEBP family members revealed that the key functional and the ligand binding sites were present, and the amino acid residues 71D, $85 \mathrm{H}, 87 \mathrm{H}, 110 \mathrm{~S}, 111 \mathrm{P}, 112 \mathrm{~K}, 118 \mathrm{H}$, and $120 \mathrm{~F}$, needed for formation of the ligand binding site, were present (Banfield and Brady, 2000) (Figure 3B). Phylogenetic analysis showed that the bamboo BoTFL1-like gene shared up to $90 \%$ identity with genes from barley, rye grass, and rice, indicating that it was highly conserved in evolutionary terms.

TFL1 is a key gene that controls inflorescence meristem characteristics throughout the life cycle; its level of expression is low in early development but increases during the transformation of the inflorescence meristem to a floral meristem (Ratcliffe et al., 1998; Kotake et al., 2003). Real-time PCR analysis showed that the level of BoTFL1-like expression was 3.5 times higher in floral buds than in vegetative buds (Figure 5), indicating that BoTFL1-like gene expression was at a low level in the vegetative stage but significantly increased in the reproductive stage. Our results suggested that the BoTFL1-like gene could play an important role in maintaining reproductive growth. Our observations are similar to a previous study that showed that the TFL1 gene is expressed at a low level in early development and could inhibit vegetative growth from switching to reproductive growth, and that a higher level of expression could maintain the properties of the inflorescence meristem during flower development (Shannon and Meeks-Wagner, 1991).

Overexpression of the BoTFL1-like gene in 35S::BoTFL1-like transgenic Arabidopsis plants caused a significant delay in flowering and a change in inflorescence structure compared to the wild type. This demonstrated that BoTFL1-like genes could have a role in the maintenance of vegetative growth and in delaying the phase transition from vegetative growth to reproductive growth in Arabidopsis. The $35 S: \because O S C E N 1$ and $35 S: \because O S C E N 2$ transgenic Arabidopsis plants flowered much later and showed increased branching inflorescence architectures, a similar phenotype as 35S::TFL1 transgenic Arabidopsis (Nakagawa et al., 2002; Zhang et al., 2005). Transgenic Arabidopsis plants overexpressing the ryegrass TFL1 homolog LPTFL1 also have the same phenotype as 35S::TFL1 transgenic Arabidopsis plants, with delayed flowering and a dramatic change in structure such as extensive lateral branching and increased growth of all vegetative organs (Jensen et al., 2001). Therefore, the transgenic Arabidopsis plants 35S::BoTFL1-like, 35S::OsCEN1, 35S::OsCEN2 and 35S::LpTFL1 share a similar effect of delayed flowering and altered inflorescence structure or morphology. One possible mechanism for these effects is that the transgenes disrupt the normal function of the endogenous TFL1 gene by competing for binding sites to specific transcription factors because they all have similar binding sites. Investigation of this possibility is under way.

The complementation experiment demonstrated that 35 S::BoTFL1-like was able to almost fully restore the phenotype of the tfll-13 mutant. As shown in Figure 7A, at the vegetative stage, the tfl1-13 mutant is reduced in size compared to the wild type but overexpression of BoTFL1-like cDNA significantly delayed terminal flower development and rescued the height phenotype, although it did not affect the number of rosette inflorescences. Moreover, the abnormal mutant flower structure was also restored (Figure 7B and C). Like 35S::BoTFL1like, 35S::LpTFL1 also rescued the phenotype of the severe tfl-14 mutant of Arabidopsis 
(Jensen et al., 2001). This suggested that BoTFL1-like may have a similar function to that of TFL1 in Arabidopsis, in regulating or controlling the time of flowering.

\section{Conflicts of interest}

The authors declare no conflict of interest.

\section{ACKNOWLEDGMENTS}

We thank Prof. Lichun Huang (Institute of Plant and Microbial Biology, Academia Sinica, Taiwan, China) for providing tissue culture plants of B. oldhami. Research supported by the Ministry of Science and Technology of China (grant \#2012CB723008), the Top Key Discipline of Forestry of Zhejiang Province (grant \#KF201304), and National the Natural Science Foundation of China (grant \#31270715 and \#31000295).

\section{REFERENCES}

Abdullah MP, Ab Shukor NA, Tahery Y, Norlia B, et al. (2009). Terminal flower 1 (TFL1) homolog genes in monocots. Eur. J. Sci. Res. 38: 26-37.

Argiriou A, Michailidis G and Tsaftaris AS (2008). Characterization and expression analysis of TERMINAL FLOWERI homologs from cultivated allotetraploid cotton (Gossypium hirsutum) and its diploid progenitors. J. Plant Physiol. 165: 1636-1646.

Banfield MJ and Brady RL (2000). The structure of antirrhinum centroradialis protein (CEN) suggests a role as a kinase regulator. J. Mol. Biol. 297: 1159-1170.

Banfield MJ, Barker JJ, Perry AC and Brady RL (1998). Function from structure? The crystal structure of human phosphatidylethanolamine-binding protein suggests a role in membrane signal transduction. Structure 6: 1245-1254.

Bowman JL, Alvarez J, Weigel D, Meyerowitz EM, et al. (1993). Control of flower development in Arabidopsis thaliana by APETALA1 and interacting genes. Development 119: 721-743.

Clough SJ and Bent AF (1998). Floral dip: a simplified method for Agrobacterium-mediated transformation of Arabidopsis thaliana. Plant J. 16: 735-743.

Daniel H and Janzen (1976). Why bamboos wait so long to flower. Annu. Rev. Ecol. Syst. 7: 347-391.

Esumi T, Kitamura Y, Hagihara C, Yamane H, et al. (2010). Identification of a TFL1 ortholog in Japanese apricot (Prunus mume Sieb. et Zucc.). Sci. Hortic. 125: 608-616.

Franklin DC (2004). Synchrony and asynchrony: observations and hypotheses for the flowering wave in a long-lived semelparous bamboo. J. Biogeogr. 31: 773-786.

Gielis J, Goetghebeur P and Debergh P (1999). Physiological aspects and experimental reversion of flowering in Fargesia murieliae (Poaceae, Bambusoideae). Syst. Geogr. Plants 68: 147-158.

Guo XZ, Zhao Z, Chen JH, Hu XH, et al. (2006). A putative CENTRORADIALIS/TERMINAL FLOWER 1-like gene, Ljcen1, plays a role in phase transition in Lotus japonicas. J. Plant Physiol. 163: 436-444.

Hayama R and Coupland G (2004). The molecular basis of diversity in the photoperiodic flowering responses of Arabidopsis and rice. Plant Physiol. 135: 677-684.

Hengst U, Albrecht H, Hess D and Monard D (2001). The phosphatidylethanolamine binding protein is the prototype of a novel family of serine protease inhibitors. J. Biol. Chem. 276: 535-540.

Hsu YH, Annamalai P, Lin CS, Chen YY et al. (2000). A sensitive method for detecting bamboo mosaic virus (BaMV) and establishment of BaMV-free meristem-tip cultures. Plant Pathol. 49: 101-107.

Jensen CS, Salchert K and Nielsen KK (2001). A terminal flowerl-like gene from perennial ryegrass involved in floral transition and axillary meristem identity. Plant Physiol. 125: 1517-1528.

Kikuchi R, Kawahigashi H, Ando T, Tonooka T, et al. (2009). Molecular and functional characterization of PEBP genes in barley reveal the diversification of their roles in flowering. Plant Physiol. 149: 1341-1353.

Kobayashi Y, Kaya H, Goto K, Lwabuchi M, et al. (1999). A pair of related genes with antagonistic roles in mediating flowering signals. Science 286: 1960-1962.

Kotake T, Takada S, Nakahigashi K, Ohto M, et al. (2003). Arabidopsis TERMINAL FLOWER 2 gene encodes a 
heterochromatin protein 1 homolog and represses both FLOWERING LOCUS T to regulate flowering time and several floral homeotic genes. Plant Cell Physiol. 44: 555-564.

Kotoda N, Iwanami H, Takahashi S and Abe K (2006). Antisense expression of MdTFL1, a TFL1-like gene, reduces the juvenile phase in apple. J. Am. Hortic. Sci. 131: 74-81.

Liljegren SJ, Gustafson-Brown C, Pinyopich A, Ditta GS, et al. (1999). Interactions among APETALA1, LEAFY, and TERMINAL FLOWER1 specify meristem fate. Plant Cell 11: 1007-1018.

Lin EP, Peng HZ, and Jin QY (2009). Identification and characterization of two bamboo (Phyllostachys praecox) AP1/ SQUA-like MADS-box genes during floral transition. Planta 231: 109-120.

Lin XC, Chow TY, Chen HH, Liu CC, et al. (2010). Understanding bamboo flowering based on large-scale analysis of expressed sequence tags. Genet. Mol. Res. 9: 1085-1093.

Lynn JP, Carol JL and Linda LW (2004). Isolation and characterization of a TERMINAL FLOWER homolog and its correlation with juvenility in Citrus. Plant Physiol. 135: 1540-1551.

Nakagawa M, Shimamoto K and Kyozuka J (2002). Overexpression of $R C N 1$ and $R C N 2$, rice Terminal Flower1/Centroradialis homologs, confers delay of phase transition and altered panicle morphology in rice. Plant J. 29: 743-750.

Ohshima S, Murata M, Sakamoto W, Ogura Y, et al. (1997). Cloning and molecular analysis of the Arabidopsis gene Terminal Flower 1. Mol. Genet. Genomics 254: 186-194.

Parcy F, Bomblies K and Weigel D (2002). Interaction of LEAFY, AGAMOUS and TERMINAL FLOWER1 in maintaining floral meristem identity in Arabidopsis. Development 129: 2519-2520.

Peng Z, Lu Y, Li L, Zhao Q, et al. (2013). The draft genome of the fast-growing non-timber forest species moso bamboo (Phyllostachys heterocycla). Nat. Genet. 45: 456-461.

Ratcliffe OJ, Amaya I, Vincent CA, Rothstein S, et al. (1998). A common mechanism controls the life cycle and architecture of plants. Development 125: 1609-1615.

Ratcliffe OJ, Bradley DJ and Coen ES (1999). Separation of shoot and floral identity in Arabidopsis. Development 126: 1109-1120.

Sato H, Heang D, Sassa H and Koba T (2009). Identification and characterization of FT/TFL1 gene family in cucumber. Breed. Sci. 59: 3-11.

Schultz EA and Haughn GW (1993). Genetic analysis of the floral initiation process (FLIP) in Arabidopsis. Development 119: 745-765.

Shannon S and Meeks-Wagner DR (1991). A mutation in the Arabidopsis TFL1 gene affects inflorescence meristem development. Plant Cell 3: 877-892.

Tahery YH, Abdul-Hamid H, Tahery E, Deljoo E, et al. (2011). Terminal Flower 1 (TFL1) homolog genes in dicot plants. World Appl. Sci. J. 12: 545-551.

Tian B, Chen Y, Yan Y and Li D (2005). Isolation and ectopic expression of a bamboo MADS-box gene. Chin. Sci. Bull. 50: 217-224.

Tian B, Chen Y, Li D and Yan Y (2006). Cloning and characterization of a bamboo Leafy Hull Sterilel homologous gene. DNA Seq. 17: 143-151.

Wang RH, Albani MC, Vincent C, Bergonzi S, et al. (2011). Aa TFL1 confers an age-dependent response to vernalization in perennial Arabis alpina. Plant Cell 23: 1307-1321.

Xi WY, Liu C, Hou XL and Yu H (2010). MOTHER OF FT AND TFL1 regulates seed germination through a negative feedback loop modulating ABA signaling in Arabidopsis. Plant Cell 22: 1733-1748.

Zhang SH, Hu W, Wang L, Lin C, et al. (2005). TFL1/CEN-like genes control intercalary meristem activity and phase transition in rice. Plant Sci. 168: 1393-1408. 